\title{
Application of Conventional Techniques for the Diagnosis of Staphylococcus lugdunensis in Clinical Microbiology
}

\author{
Leonor Aties López ", Milagros de la Caridad Milá Pascual, José Antúnez Coca \\ Diagnostic Media Department, Faculty of Nursing Health Technology, Medical Sciences University, Santiago de Cuba, Cuba \\ Email address: \\ leonoraties@infomed.sld.cu(L. A. López),milagros.milá@infomed.sld.cu(M. de la C. M. Pascual), \\ jose.antunez@infomed.sld.cu(J.A. Coca) \\ *Corresponding author
}

\section{To cite this article:}

Leonor Aties López, Milagros de la Caridad Milá Pascual, José Antúnez Coca. Application of Conventional Techniques for the Diagnosis of Staphylococcus lugdunensis in Clinical Microbiology. Cell Biology. Vol. 9, No. 1, 2021, pp. 16-19. doi: 10.11648/j.cb.20210901.13

Received: March 17, 2021; Accepted: April 15, 2021; Published: April 29, 2021

\begin{abstract}
Introduction: the participation of coagulase negative staphylococci in human diseases is an important problem, particularly in hospital settings where these species often act as opportunistic pathogens. Objective: to show the results obtained after applying conventional techniques for its detection. Results: the tests Cumpling factor, Detection of hemolysin $\delta$-like, acidification of the substrates trehalose, mannose, mannitol and inositol were applied, as well as the ornithine decarboxylation biochemical test and the behavior of antimicrobial sensitivity was taken into account. all being feasible for the diagnosis of this microorganism due to the results obtained. Conclusions: Staphylococcus lugdunensis is an atypical SCoN compared to the rest of the SCoNs, both due to its special virulence characteristics and its striking sensitivity to multiple antimicrobials, including penicillin sensitivity in many cases. Its diagnosis in clinical microbiology laboratories is simple, given the suspicion of this species by the positive compliance factor test, a complete identification of it must be carried out through the decarboxylation of ornithine, the acidification of mannose, as well as antimicrobial sensitivity, are useful tests to differentiate this microorganism from the rest of its species.
\end{abstract}

Keywords: Staphylococcus lugdunensis, Microbiological Diagnosis, Virulence Factor

\section{Introduction}

Coagulase negative staphylococci (SCN) comprise a large number of different species of Staphylococcus aureus, named for their inability to coagulate plasma due to lack of coagulase enzyme production. Traditionally, they were considered innocuous or little virulent opportunistic pathogens when found as commensals on the skin, but with the advancement of medicine and the use of invasive diagnostic and therapeutic methods, coagulase-negative staphylococci have become first-order pathogens. [1]

The clinical importance of coagulase negative staphylococci as etiological agents of healthcare-related infections has been increasing in recent decades, due to the ability of these microorganisms to adhere and form biofilms on the surface of intravenous devices and catheters assuming a important role in the pathogenesis of these infections. [2]

The clinical manifestations caused by these agents can be mild and nonspecific, often without signs of systemic involvement. Thus, for example, mortality from coagulase negative staphylococcal bacteremia is low, especially if it is treated quickly and correctly; however, these microorganisms can be the cause of severe sepsis and septic shock in immunosuppressed patients, or if certain species of coagulase negative staphylococcus possessing certain virulence factors are involved, such as Staphylococcus lugdunensis. [3]

It has emerged as an agent with remarkable clinical and microbiological characteristics that stand out from the other coagulase negative staphylococci. Although Staphylococcus lugdunensis does not cause infection as often as Staphylococcus aureus or Staphylococcus epidermidis, its pathogenic potential should not be underestimated and it has been associated, more frequently, with a broad spectrum of milder infections, such as skin and tissue infections. soft (cellulitis, subcutaneous abscesses, mastitis) and those of bones and joints (chronic osteomyelitis, arthritis). [4, 5]

It has also been described as a causal agent of bacteremia, infections associated with catheters and intravascular 
prostheses, infections of the central nervous system, peritonitis associated with ambulatory peritoneal dialysis, urinary tract infections, postsurgical endophthalmitis, ocular, otic, oral infections and urinary tract infection, among other. The Staphylococcus lugdunensis species was described by Freney et al [1] in 1988; the term lugdunensis derives from Lugdunum, the Latin name for Lyon, the city where isolated for the first time. The aforementioned species is part of the microbiota of the skin and it is also a rare but not rare human pathogen. Although it has been described with more frequent in patients with underlying diseases or in those who receive immunosuppressive therapies, it can also cause superficial infections or deep in healthy individuals. [6]

These discrepancies between the virulence of Staphylococcus lugdunensis and that of other coagulasenegative staphylococci must be added a surprising characteristic of this species, which is that most strains are sensitive to a large number of antimicrobials. The relative absence of diversity

Genetics observed among the different strains of this species and the high prevalence of sensitivity to multiple antimicrobial agents suggests that the evolution of Staphylococcus lugdunensis has occurred by a different pathway from that of other pathogenic coagulase-negative staphylococcal species. Given these differences, whenever Staphylococcus lugdunensis is isolated from clinical samples, it should be thought of as a pathogen, and only occasionally can it be considered as a contaminant or colonizer. [1-3]

For this reason, the authors believe that an active search for this microorganism is necessary, since identification at the species level is not a common practice in microbiology laboratories, and it can also be confused with Staphylococcus aureus on some occasions due to production of the affinity factor for fibrinogen or clumping factor, however it should be noted that the reaction produced by Staphylococcus lugdunensis is later and weaker than that produced by Staphylococcus aureus. [6, 7]

Taking into account the above, it is the purpose of this communication to disclose the results obtained after applying conventional techniques for the diagnosis of Staphylococcus lugdunensis in the Laboratory of Clinical Microbiology.

\section{Developing}

\subsection{Clinical and Interpretation Criteria}

The Infectious Diseases Society of America (IDSA) was the first to consider S. lugdunensis as a different CoNS in its 2015 guidelines for the management of the diagnosis of osteomyelitis in adults The IDSA does not recommend a bone biopsy in patients with suspected osteomyelitis when infection with $\mathrm{S}$. aureus or $\mathrm{S}$. lugdunensis has been established based on positive blood cultures. In the same year, the European Society of Cardiology published guidelines for the management of endocarditis and emphasized the role of S. lugdunensis in destructive infective endocarditis, unlike other CoNS. These results are clearly supported by growing clinical evidence, and the causal role of the virulence factors described in this review is likely, even if in vitro and animal models are lacking to model this relationship. At the same time, the European Manual of Clinical Microbiology still warns that two CoNS positive samples are required in a clinical sample to be considered significant, because contamination and colonization remain frequent. [13-15] On the contrary, only a positive sample for S. aureus in blood cultures or a bone sample is sufficient to be considered pathological. Clinical and microbiological evidence is now sufficiently concordant to consider that $\mathrm{S}$. lugdunensis cannot be considered a regular CoNS. [1] showed in a retrospective analysis that $45 \%$ of 29 patients with a single positive blood culture for $\mathrm{S}$. lugdunensis did have bacteremia [7]. The authors used the criteria published by Souvenir et al. [8] that have been shown to be useful in determining the clinical significance of blood culture positivity for CoNS. Regarding bone, joint, and prosthetic joint infections, the most informative data came from the VISLISI clinical trial. [8] Among 28 patients with proven infections, $25 \%$ had only one positive sample. In this trial, infection was considered to have been proven in cases with a single positive sample if three criteria were met:

1. The existence of clinical signs of infection

2. Growing a pure culture

3. The sample that was not taken from a known niche for this microorganism

Therefore, it is proposed that $\mathrm{S}$. lugdunensis be considered a pathogen in deep clinical, samples, such as blood cultures or bone and joint samples, even if only one sample is positive but in pure culture, at least until any other diagnosis has been verified. However, it is advisable to exercise caution when interpreting single positive samples in skin and soft tissues or within a known niche of this bacterium such as the inguinal, axillary and nasal region. [8]

\subsection{Virulence Factors}

The virulence of Staphylococcus lugdunensis is due to multiple mechanisms, including which is the production of adherent surface proteins, which allows to bind to host tissues and proteins, as well as to surfaces of foreign bodies. [1] Its ability to bind fibrinogen, fibronectin, and Von Willebrand factor (glycoprotein involved in coagulation) by

vWf-binding protein may contribute to its success in producing endocarditis. [13, 15] Another virulence mechanism used by some Staphylococcus strains lugdunensis is the formation of biofilm, which presents important differences with compared to other coagulase negative staphylococci in terms of the composition of the biofilm matrix and the genomic organization of the ICA locus, which serves as example of the differential characteristics that this microorganism has acquired during its evolution. In addition, Staphylococcus lugdunensis is resistant to high lysozyme concentrations and produces a heat stable $\delta$-like hemolysin and similar to the $\delta$-hemolysin of Staphylococcus aureus; $[13,14]$ this hemolysin produces synergy with Staphylococcus aureus $\beta$-hemolysin, a characteristic that 
allows the detection of its presence in the laboratory by observing the synergy phenotype hemolytic (complete hemolysis of red blood cells) in an approximation test of the 2 species on blood agar medium.

In Staphylococcus aureus, $\delta$-toxin is encoded by the hld gene, and in Staphylococcus lugdunensis has been shown to have a sequence similar to that of hld gene, encoded at a locus called slush (Staphylococcus lugdunensis synergistic hemolysin). [14, 15] The presence of sequences related to the accessory regulatory gene (agr) of the Staphylococcus aureus in the Staphylococcus lugdunensis genome contributes also to the virulence of this microorganism. Staphylococcal agr is a system of autoinduction that acts as a global regulator of virulence factors, particularly exoproteins, including enterotoxins, hemolysins, and numerous host protein modifying enzymes (lipases, esterases, proteases). In the Staphylococcus lugdunensis homologous regions have been identified with the agr of the Staphylococcus aureus, which are very actively transcribed. Regulation transcriptional locus slush is probably also under the control of the locus Agr. [14]

\section{Results}

The great virulence and destructive nature of Staphylococcus lugdunensis are sufficient reasons for the rapid identification of this microorganism at the species level, when its presence is suspected in an infection and especially it is isolated from a sterile site. [1]

Despite these characteristics, Staphylococcus lugdunensis is a species that can be identified quite easily in clinical microbiology laboratories. Authors such as Argemi X et al [8] highlight in their article the obsolescence of the techniques described, however due to the The possibilities that the laboratories have have been useful for its diagnosis, being confirmed in the reference laboratories. For which the decarboxylation of ornithine and the characteristics of the culture were used as an important parameter. [2-4]

Regarding the characteristics of the culture, it should be noted that Staphylococcus lugdunensis usually manifests as a generally heterogeneous culture, with different colony sizes, and different expression of $\beta$-hemolysis; the extension of the incubation time to $48-72 \mathrm{~h}$ allows to observe much more homogeneous colonies that present a more typical appearance; these colonies are yellowish-white, creamy, and with a small halo of $\beta$-hemolysis (unlike the broader $\beta$ hemolysis of S. aureus). [9-13]

Related to the production of biofilms, the authors agree with what was obtained by other researchers, $[11,12]$ since there was a biofilm production within $6 \mathrm{~h}$ after culture and highly consistent not appreciated in other staphylococci. Concerning biofilm production it should be noted, bacteria can respond rapidly to unfavorable or stressful environmental conditions by reducing their metabolic activity, altering their gene expression or inducing changes, entering a state of torpor. Biofilms themselves are an example of a bacterium under stress, due to the lack of nutrients and oxygen, this condition is characterized by the presence of heterogeneous cells, with different physiological states, whose emergence depends on the microenvironmental conditions in their environment, Therefore, access to nutrients and oxygen in the deep layers of biofilms is more unfavorable than in the upper layers, variant subpopulations of cells can emerge, changing their growth mode to promote their survival in stressed or limited environmental conditions, calling themselves to this quality persistence as a tolerance mechanism. [8]

Together, the acidification of the substrates trehalose, mannitol, inositol and mannose was carried out, the latter contributes to a certainty in the diagnosis as a result of a definitive differentiation. The strains of $S$. lugdunensis use this sugar while those of $S$. haemolyticus do not. After performing the tests, the following results were obtained

Observe in (figure 1) the positive tube on the left, the purple color indicates that ornithine decarboxylation occurred, while the negative, is perceived by the yellow color, a test that is required for a correct identification of this species, since it is its main biochemical characteristic. $[9,10]$

In (figure 2) the appearance of $\delta$-like hemolysin is observed, since the production of synergy with the $\beta$ hemolysis of Staphylococcus aureus occurred. (Figure 3) shows the presence of agglutination (lumps), indicating the production of the compliance factor or affinity factor for fibrinogen. Unlike S. aureus, S. lugdunensis does not have free coagulase (negative tube coagulase test), but $60-80 \%$ of strains produce a membrane-bound form of the enzyme (the so-called clumping factor) that gives a positive result in both the human plasma slide coagulase test and commercial rapid latex agglutination tests, which can lead to identification errors in the laboratory. However, it should be noted that the reaction produced by $\mathrm{S}$. lugdunensis is later and weaker than that produced by S. aureus. [8-14]

Although the sensitivity of coagulase-negative staphylococci to antibiotics is unpredictable, and depends mainly on the species and the origin of the isolated strain (hospital or out-ofhospital environment). The usual, to date, in S. lugdunensis is its sensitivity to all groups of antibiotics used in the treatment of staphylococcal infections, including penicillins, which is consistent with the literature consulted, [8-12] Since similar results were obtained in this study, as the production of $\beta$ lactamase was negative, the isolated strains were sensitive to most of the antimicrobials used, including penicillins.



Figure 1. Decarboxylation of ornithine. 


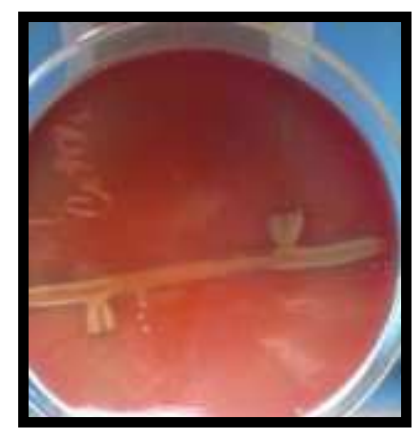

Figure 2. Detection of hemolysin $\delta$-like.

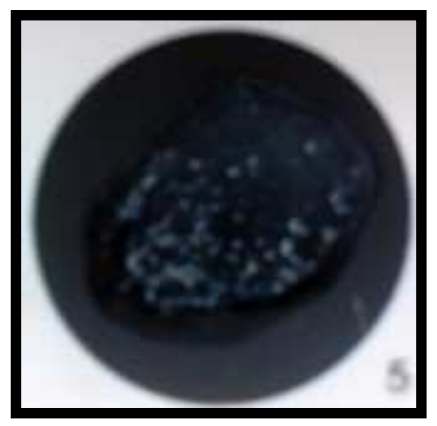

Figure 3. Cumpling factor.

\section{Conclusions}

Staphylococcus lugdunensis is an atypical SCoN compared to the rest of the SCoNs, both due to its special virulence characteristics and its striking sensitivity to multiple antimicrobials, including penicillin sensitivity in many cases. Its diagnosis in clinical microbiology laboratories is simple, given the suspicion of this species by the positive compliance factor test, a complete identification of it must be carried out through the decarboxylation of ornithine, the acidification of mannose, as well as antimicrobial sensitivity, are useful tests to differentiate this microorganism from the rest of its species.

\section{References}

[1] Aties López Leonor, Moya Jústiz Gilberto, Antúnez Coca José, Milá Pascual Milagros de la Caridad. Significación clínica y aspectos microbiológicos para el diagnóstico de pacientes con Staphylococcus lugdunensis. MEDISAN [Internet]. $2020 \mathrm{Abr}$ [citado $2021 \mathrm{Abr}$ 09]; 24 (2): 303-311. Disponible en:

http://scielo.sld.cu/scielo.php?script=sci_arttext\&pid=S102930192020000200303\&lng=es. Epub 24-Äbr-2020.

[2] Halaji M, Karimi A, Shoaei P, Nahaei M, Khorvash F, Ataei B et al. Distribution of SCCmec Elements and Presence of Panton-Valentine Leukocidin in Methicillin-Resistant Staphylococcus epidermidis Isolated from Clinical Samples in a University Hospital of Isfahan City, Iran. J Clin Diagn Res. 2017 Jul; $11 \quad$ (7): DC27-DC31. doi: 10.7860/JCDR/2017/25518.10258. Epub 2017 Jul 1. PubMed PMID: 28892893; PubMed Central PMCID: PMC5583865.

[3] Palavecino EL. Rapid MethodsforDetection of MRSA in ClinicalSpecimens. Methods Mol Biol. 2020; 2069: 29-45. doi: 10.1007/978-1-4939-9849-4_2. PMID: 31523763.
[4] Karakullukçu A, Kuşkucu MA, Ergin S, Aygün G, Midilli K, Küçükbasmaci Ö. Determination of clinicalsignificance of coagulase-negativestaphylococci in blood cultures. Diagn Microbiol Infect Dis. 2017 Mar; 87 (3): 291-294. doi: 10.1016/j.diagmicrobio.2016.12.006. Epub 2016 Dec 14. PMID: 28012637.

[5] Argemi X, Hansmann Y, Riegel P, Prévost G. Is Staphylococcus lugdunensis significant in clinical samples? J Clin Microbiol. 2017 Aug 23. pii: JCM.00846-17. doi: 10.1128/JCM.00846-17. [Epub ahead of print] PubMed PMID: 28835477.

[6] França A, Gaio V, Lopes N, Melo LDR. Virulence Factors in Coagulase-Negative Staphylococci. Pathogens. 2021 Feb 4; 10 (2): 170. doi: 10.3390/pathogens10020170. PMID: 33557202; PMCID: PMC7913919.

[7] McHardy IH, Veltman J, Hindler J, Bruxvoort K, Carvalho MM, Humphries RM. Clinical and Microbiological Aspects of $\beta$-Lactam Resistance in Staphylococcus lugdunensis. J Clin Microbiol. 2017 Feb; 55 (2): 585-595. doi: 10.1128/JCM.02092-16. Epub 2016 Dec 7. PMID: 27927926; PMCID: PMC5277529.

[8] Taha L, Stegger M, Söderquist B. Staphylococcus lugdunensis: antimicrobial susceptibility and optimal treatment options. Eur J Clin Microbiol Infect Dis. 2019 Aug; 38 (8): 1449-1455. doi: 10.1007/s10096-019-03571-6. Epub 2019 May 29. PMID: 31144243; PMCID: PMC6647525.

[9] Parthasarathy S, Shah S, Raja Sager A, Rangan A, Durugu S. Staphylococcus lugdunensis: Review of Epidemiology, Complications, and Treatment. Cureus. 2020 Jun 24; 12 (6): e8801. doi: 10.7759/cureus.8801. PMID: 32617242; PMCID: PMC7325404.

[10] Heldt Manica LA, Cohen PR. Staphylococcus lugdunensis Infections of the Skin and Soft Tissue: A Case Series and Review. Dermatol Ther (Heidelb). 2017Oct 11. doi: 10.1007/s13555-017-0202-5. [Epubahead of print] PubMed PMID: 29022273.

[11] Lindsay JA. Staphylococci: Evolving Genomes. Microbiol Spectr. 2019 Nov; 7 (6). doi: 10.1128/microbiolspec.GPP30071-2019. PMID: 31845645.

[12] Dahyot S, Oxaran V, Niepceron M, Dupart E, Legris S, Destruel L, Didi J, Clamens T, Lesouhaitier O, Zerdoumi Y, Flaman JM, Pestel-Caron M. Role of the LytSR TwoComponent Regulatory System in Staphylococcus lugdunensis Biofilm Formation and Pathogenesis. Front Microbiol. 2020 Jan 24; 11: 39. doi: 10.3389/fmicb.2020.00039. PMID: 32038604; PMCID: PMC6993578.

[13] Al Majid FM. Staphylococcus lugdunensis from gluteal abscess to destructive native triple valve endocarditis. Saudi Med J. 2018 Oct; 39 (10): 1050-1053. doi: 10.15537/smj.2018.10.22905. PMID: 30284590; PMCID: PMC6201034.

[14] Heilbronner S, Foster TJ. Staphylococcus lugdunensis: a Skin Commensal with Invasive Pathogenic Potential. Clin Microbiol Rev. 2020 Dec 23; 34 (2): e00205-20. doi: 10.1128/CMR.00205-20. PMID: 33361142; PMCID: PMC7950365.

[15] Natsis NE, Cohen PR. Coagulase-Negative Staphylococcus Skin and Soft Tissue Infections. Am J Clin Dermatol. 2018 Oct; 19 (5): 671-677. doi: 10.1007/s40257-018-0362-9. PMID: 29882122. 